\title{
The Kinematics in the Cores of Low Surface Brightness Galaxies
}

\author{
R. A. Swaters
}

Johns Hopkins University, 3400 N. Charles St., Baltimore MD 21218, U.S.A.; and Space Telescope Science Institute, 3700 San Martin Dr., Baltimore, MD 21218, U.S.A.

\author{
M. A. W. Verheijen \\ Astrophysikalisches Institut Potsdam, An der Sternwarte 16, 14482 \\ Potsdam, Germany.
}

M. A. Bershady

Astronomy Department, University of Wisconsin - Madison, 475 N. Charter St., Madison, WI 53706, U.S.A.

D. R. Andersen

Max Planck Institut für Astronomie, Königstuhl 17, 69117 Heidelberg, Germany

\begin{abstract}
Systematic effects on $\mathrm{HI}$ and $\mathrm{H} \alpha$ long-slit observations make a measurement of the inner slope of the dark matter density distribution difficult to determine. Halos with constant density cores and ones with $r^{-1}$ profiles both appear consistent with the data, although constant density cores generally provide better fits. High-resolution, two-dimensional velocity fields remove most of the systematic effects, yet as a result of noncircular and random motions the inner slopes still cannot be accurately measured. Halo concentration parameters provide a more useful test of cosmological models because they are more tightly constrained by observations. The concentration parameters for LSB galaxies appear consistent with, but on the low end of the distribution predicted by CDM.
\end{abstract}

\section{Introduction}

Studies of dark matter in spiral galaxies have been plagued by uncertainties in the mass-to-light ratio $(\mathrm{M} / \mathrm{L})$ of the stellar disk, resulting in a large degeneracy in the mass modeling (e.g., van Albada et al. 1985). This degeneracy can largely be avoided by studying dwarf and low surface brightness (LSB) galaxies. Even though the central parts of the observed rotation curves can in principle be explained by scaling up the contribution of the stellar disks (Swaters 1999; Swaters, Madore, \& Trewhella 2000), the inferred stellar M/Ls are much higher than expected from population synthesis modeling (e.g., Bell \& de Jong 2001). 
For reasonable M/Ls, these galaxies are dominated by dark matter at all radii, making dwarf and LSB galaxies ideal for studying the properties of dark matter.

The power law slopes of the central dark matter density distributions $\rho(r) \propto$ $\rho^{-\alpha}$ are of particular interest. Cosmological simulations indicate $\alpha$ depends on the nature of dark matter. A measurement of $\alpha$ may thus provide constraints on the nature of dark matter and theories of galaxy formation. Unfortunately, it is difficult to measure the inner slope $\alpha$ accurately, both from simulations (e.g., Power et al. 2003; White, this volume; Navarro, this volume), and from observations, as is discussed in detail below (see also de Blok, this volume).

\section{Systematic effects on rotation curves}

The main assumption in using rotation curves to infer the properties of dark matter halos is that the rotation velocities reflect the gravitational potential. However, observational effects, such as poor spatial resolution or slit offsets, and intrinsic properties of the galaxies, such as pressure support or noncircular motions, may affect the derived rotation curves and lead to systematic uncertainties in the derived dark matter properties.

\subsection{HI observations}

Earlier HI studies found that dwarf and LSB galaxies have slowly rising inner rotation curves (see e.g., Swaters 1999 and references therein). Because of their shallow slopes, these rotation curves were found to be inconsistent with the steep halos predicted by cold dark matter (e.g., Navarro et al. 1996; McGaugh \& de Blok 1998). More recent studies raised the concern that some of these HI rotation curves may be affected by beam smearing, and found that the rotation curves of dwarf and LSB galaxies rise more steeply when beam smearing is taken into account (Swaters 1999; Blais-Ouellette et al. 1999; Swaters et al. 2000).

However, beam smearing in itself does not constitute an insurmountable problem. It is possible to correct the measured rotation curves for the effects of beam smearing (Swaters 1999), or its effects can be incorporated in the modeling procedure (e.g., van den Bosch et al. 2000). Nonetheless, because of their relatively low resolution, HI observations may not be best suited to determine the inner rotation curve slopes. High angular resolution observations, such as $\mathrm{H} \alpha$ long slit or Fabry-Perot spectroscopy, thus seem more suited.

\subsection{H $\alpha$ long-slit observations}

Despite the high spatial resolution of $\mathrm{H} \alpha$ long-slit observations, it remains difficult to measure $\alpha$ accurately. Different studies find conflicting results: some find LSB galaxies to be consistent with steep inner slopes (e.g., Swaters et al. 2003a, hereafter SMvdBB), yet others find them inconsistent with steep slopes (e.g., de Blok et al. 2001a,b; de Blok \& Bosma 2002; Marchesini et al. 2002). This inconsistency remains even when identical datasets are used (SMvdBB).

This apparent discrepancy may well be the result of systematic effects on the long-slit observations, which all lead to an underestimate of the inner slope. Several effects may contribute: 1) Seeing tends to wash out the inner gradient of the rotation curve, leading to an underestimate of the inner slope. 2) If the slit is 
opened wider, parts of the galaxy with lower radial velocities will also be visible through the slit, diluting the inner gradient and resulting in an underestimate of the inner slope. 3) Slit offsets (the combined results of telescope pointing errors, errors in the coordinates of the optical centers, and offsets between optical and dynamical centers) will cause the steepest gradient in the rotation curve to be missed, leading to an underestimate of the inner slope. 4) At inclinations close to edge-on, one line of sight samples a large part of the disk, resulting in a range of observed velocities. The rotation velocity is represented by the extreme velocity along the line-of-sight. Obviously, the usual method of deriving the rotation velocity by Gaussian fits or a intensity weighted mean will result in an underestimate of the rotation velocity, which in turn leads to an underestimate in the inner slope. On top of that, extinction and the distribution of $\mathrm{H} \alpha$ (e.g., a ring) may also lead to an underestimate of the inner slope. 5) If the $\mathrm{H} \alpha$ emission is concentrated toward the edge of the slit, the derived velocities will be lower, especially near the center of the galaxy, leading to an underestimate of the inner slope. In summary, all systematic effects lead to an underestimate of the inner slope, so there clearly is an strong observational bias against finding steep slopes from long-slit observations.

\section{The inner slope: poor constraints on cosmological models}

In the literature, three principal ways of measuring the inner slope of the halo density profile have been used: inversion of the rotation curve (e.g., de Blok et al. 2001, SMvdBB), fitting a power law to the observed rotation curve (e.g., Simon et al. 2003), and fitting a general mass model to the observed rotation curve (e.g., van den Bosch et al. 2000, SMvdBB).

\subsection{Rotation curve inversion}

If one assumes the disks are dynamically insignificant, and if one assumes furthermore that the dark matter has a spherically symmetric distribution, it is possible to recover the density distribution of the dark matter from the observed rotation curve in a non-parametric way. From $\nabla^{2} \Phi=4 \pi G \rho$ and $\Phi=-G M / r$ the density distribution is given by

$$
\rho(r)=\frac{1}{4 \pi G}\left(2 \frac{v}{r} \frac{\partial v}{\partial r}+\frac{v^{2}}{r^{2}}\right) .
$$

The inner slope $\alpha$ can be measured by inverting the measured rotation curve using Eq. 1, and fitting a simple power-law $\rho \propto r^{-\alpha_{m}}$ to the density distribution inside the break radius (the radius where the density profile turns from its inner slope to its outer $r^{-2}$ slope).

In Fig. 1 we present the distribution of measured inner slopes for the galaxies in the samples of de Blok et al. (2001a), de Blok \& Bosma (2002), and SMvdBB). Overplotted on the histograms are the expected distributions of measured inner slopes based on detailed modeling of the observations, including the effects of distance, seeing, slit width, and slit alignment errors. See SMvdBB for more details on the modeling. 


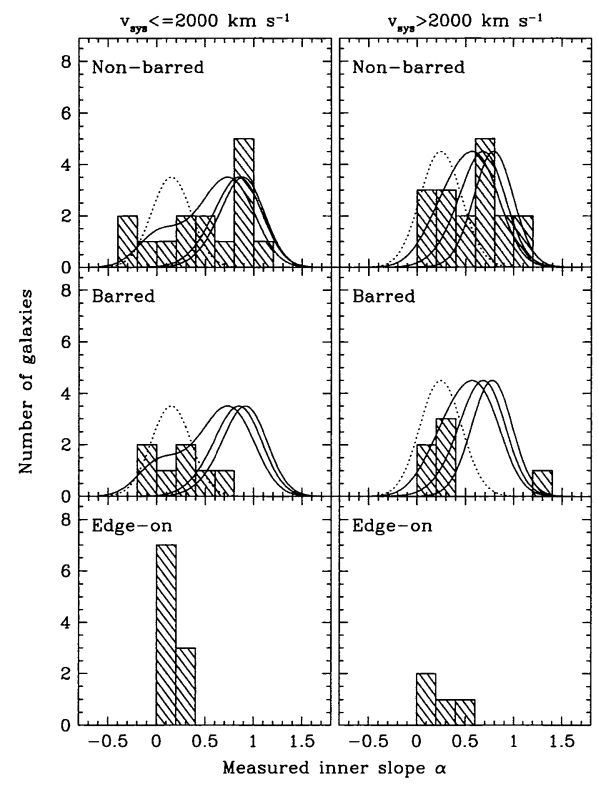

Figure 1. The distribution of measured inner slopes for the galaxies in the samples of de Blok et al. (2001a), de Blok \& Bosma (2002), and $\mathrm{SMvdBB}$ ), divided into non-barred, barred, and edge-on galaxies, both for galaxies within $2000 \mathrm{~km} \mathrm{~s}^{-1}$ (left), and for galaxies beyond $2000 \mathrm{~km} \mathrm{~s}^{-1}$. Dotted lines represent the expected distributions of inner slopes for $\alpha=0$, the solid lines from left to right give the expected distributions for $\alpha=1$, with a slit alignment error of $2^{\prime \prime}, 1^{\prime \prime}$ and $0^{\prime \prime}$.

For the non-barred galaxies the measured inner slopes span a wide range from $\alpha=0$ to $\alpha=1$. The distribution of $\alpha_{m}$ for the non-barred galaxies in the combined sample appears inconsistent with the distribution expected for halos with $\alpha=0$. However, it is possible that the stellar disks do contribute significantly and this may explain the wing to high $\alpha_{m}$ even if the halos have $\alpha=0$. The models with $\alpha=1$ appear to underpredict the number of galaxies with $\alpha_{m}$ near zero, unless the error in the slit positioning is about $2^{\prime \prime}$. An error of $2^{\prime \prime}$ in the slit positioning may not be unreasonable given the large angular size of these galaxies and their irregular, diffuse and low surface brightness nature.

Although based on only a few galaxies, the barred galaxies on average appear to have a somewhat lower $\alpha_{m}$. This may be an indication that the noncircular motions associated with the bar have affected the inner slopes. Another striking feature in Fig. 1 is the large difference in the distribution of measured inner slopes between edge-on galaxies and non-barred galaxies. This difference is most likely artificial: the method with which the rotation curves were derived lead to a severe underestimate of the inner slopes (SMvdBB).

Although the inner slope derived from rotation curve inversion has the advantage that it is a parameter-free measurement and that it directly measures the inner slope of the halo, it is particularly sensitive to systematic effects mentioned above because the inner slope is usually measured from only the innermost 

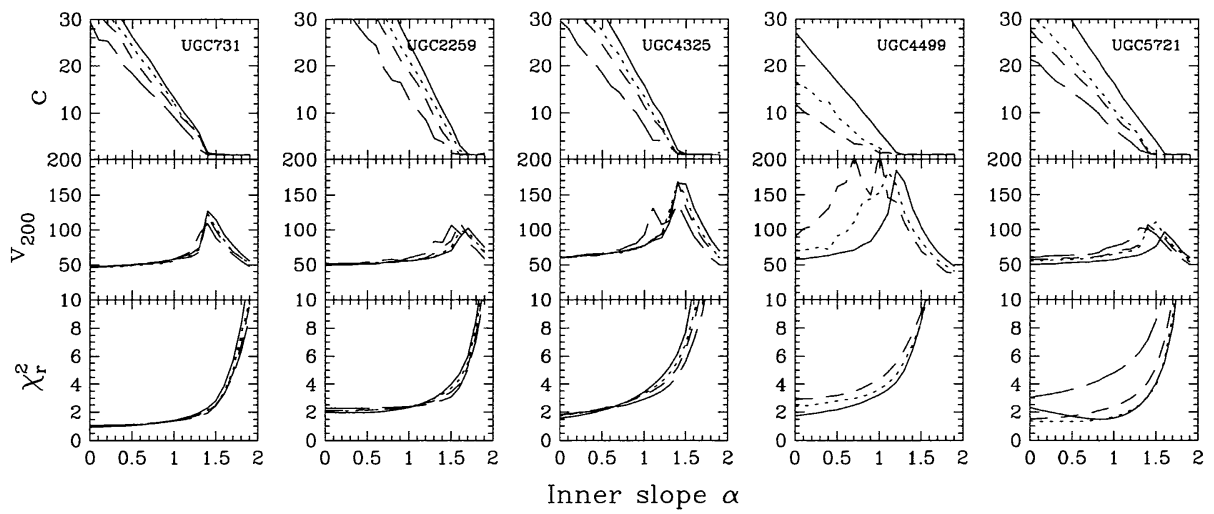

Figure 2. From top to bottom the best fit $c$, best fit $v_{200}$, and reduced $\chi^{2}$ for the generic halo fits. The solid line represents $\Upsilon_{*}=0$ (for the $R$-band), the dotted line is $\Upsilon_{*}=0.5$, the short dashed lines is $\Upsilon_{*}=1$ and the long dashed line is $\Upsilon_{*}=2$. Halos with $0<\alpha<1$ are consistent with most galaxies, although halos with $\alpha=0$ generally provide better fits.

points. As a result, it is not possible to measure the inner slopes accurately, and our conclusion is that inner slopes in the range $0<\alpha<1$ are consistent with the observations discussed here.

\subsection{Fitting a power law to the rotation curve}

Some authors have determined the inner slope by fitting a power law slope to the entire observed rotation curve (e.g. Simon et al. 2003). Although this gives a more accurate measurement than rotation curve inversion, it seems unlikely that an inner slope derived from a fit to the entire rotation curve can be directly compared to the numerical simulations, because in the simulations the slopes change with radius. A measurement of the true inner slope could be derived by fitting a power law slope to the inner parts of the rotation curve only, but that would make the measurement sensitive to the systematic effects described in the previous subsection.

\subsection{Fitting a general density profile to the rotation curve}

The inner slope can also be measured by fitting a halo model to the rotation curve based on a general density profile of the form:

$$
\rho(r)=\frac{\rho_{0}}{\left(r / r_{s}\right)^{\alpha}\left(1+r / r_{s}\right)^{3-\alpha}}
$$

where $\rho_{0}$ is the central dark matter density, and $r_{s}$ a scale radius. This density distribution changes from $\rho \propto r^{-\alpha}$ for $r \ll r_{s}$ to $\rho \propto r^{-3}$ for $r \gg r_{s}$. For $\alpha=0$ the density profile thus has a constant density core and becomes comparable to that of the pseudo-isothermal sphere, while for $\alpha=1$ it reduces to the NFW profile (Navarro et al. 1997). 


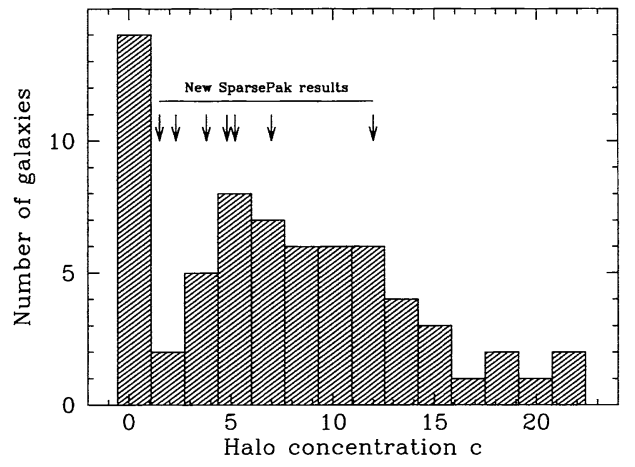

Figure 3. The shaded histogram gives the distribution of best fit $c$ parameters for galaxies in the samples of de Blok et al. (2001a), de Blok \& Bosma (2002), and SMvdBB. The arrows indicate the best fit $c$ parameters derived from new SparsePak data (Swaters et al. 2003b, Swaters et al. in preparation).

The density and scale radius derived from such mass models are less sensitive to the effects of seeing, slit width and slit offsets, because the entire rotation curve is used to determine the parameters, not only the innermost part. Consequently, these mass models are not very sensitive to the inner slope of the dark matter halo, and for most galaxies we find that mass models based on $\alpha=1$ provide fits of similar quality as those based on $\alpha=0$ (see examples in Fig. 2). This is not only true if the contribution of the stellar component is ignored, but also for models in which the stellar component plays a modest role.

From these general mass models we find that for most galaxies the inner slope is unconstrained, and fits of similar quality are obtained for the range $0<\alpha<1$, although $\alpha=0$ is somewhat preferred. Inner slopes as steep as $\alpha=1.5$ are clearly ruled out.

\section{Halo concentration: useful constraints on cosmological models}

A different way of testing the predictions of CDM simulations is through the halo concentration parameter $c$. For an NFW halo (Eq. 2 with $\alpha=1$ ), $c=$ $r_{200} / r_{s}$, where $r_{200}$ is the virial radius. An important advantage of using the halo concentration parameter is that it is less sensitive to systematic effects because it is derived from a fit to the rotation curve as a whole. The expected values for $c$ depend on the assumed cosmological parameters. For a $\Lambda$ CDM cosmology, expected values have a $2 \sigma$ range from 5 to 25, with an average of around 10 to 15 (Navarro et al. 1997, Bullock et al. 2001). In Fig. 3 the distribution of best fit $c$ values is shown; the uncertainty on $c$ from rotation curve fits is about $20 \%$ to $40 \%$ for most galaxies. The peak near $c=1$ is likely to be artificial, resulting predominantly from galaxies in which the fit is unconstrained, such as galaxies with rotation curves that extend less than two disk scale lengths, and from edge-on galaxies with possibly incorrect rotation curves. The rest of the 


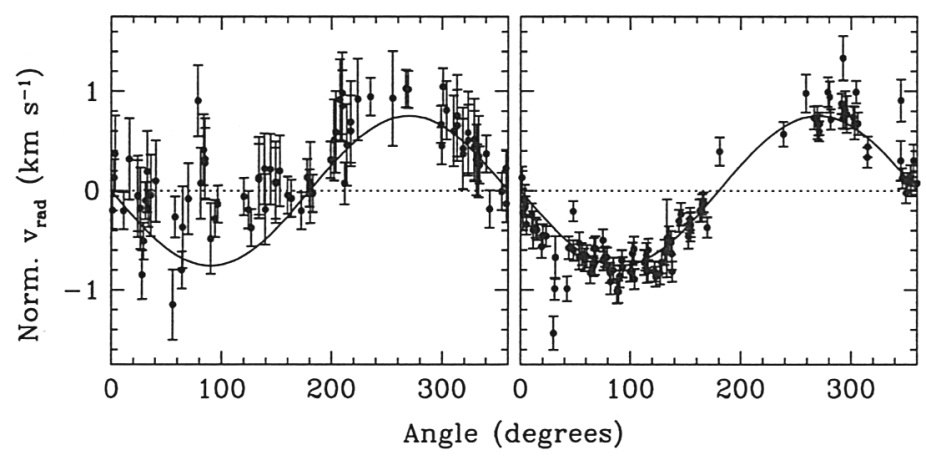

Figure 4. Radial velocity relative to the systemic velocity, normalized by rotation velocity, as function of angle in the plane, for DDO 39 (obtained with SparsePak on the WIYN telescope, Swaters et al. 2003b). The left panel shows points within $r<1 \mathrm{kpc}$, the right one points between $1.5<r<2.5 \mathrm{kpc}$. The solid line represents circular rotation.

distribution shows a large spread, and seems somewhat skewed toward smaller values of $c$ than expected in $\Lambda$ CDM.

Low values for $c$ do not necessarily indicate an inconsistency with $\Lambda$ CDM. For example, a bias toward low $c$ values in LSB galaxies could be explained if LSB galaxies preferentially form in low density halos. In addition, the concentration parameter also depends on the slope of the power spectrum of density fluctuations, and $c \sim 5$ is in agreement with models in which structure formation on small scales is suppressed (Zentner \& Bullock 2002).

\section{High Resolution, Two-Dimensional Velocity Fields}

To avoid systematic effects of long-slit and HI observations, and to map possible non-circular motions, high spatial resolution, two-dimensional velocity fields are needed. Several such studies are now available (e.g., Blais-Ouellette et al. 2001; Bolatto et al. 2002; Swaters et al. 2003b; Simon et al. 2003).

Swaters et al. (2003b) studied the kinematics in the core of the LSB galaxy DDO 39 with the SparsePak integral field spectrograph on the WIYN telescope (Bershady et al., submitted), and found random motions with an average amplitude of about $8 \mathrm{~km} \mathrm{~s}^{-1}$ throughout the galaxy, and in addition detected distinct noncircular motions as can be seen in Fig. 4. We (Swaters et al. in preparation) have obtained data for an additional 6 LSB galaxies with SparsePak. Preliminary analysis of these data again reveal significant random and noncircular motions. The origin of either component is unclear, but they cause inner slopes in the range $0<\alpha<1$ to be consistent with the data. Therefore, even though these data are a significant improvement on previous $\mathrm{HI}$ or $\mathrm{H} \alpha$ long-slit data, the inner rotation curve, and hence the inner slope of the dark matter distribution, is still uncertain due to significant noncircular and random motions.

Fortunately, the availability of two-dimensional velocity fields makes it possible to determine the kinematic center and estimate the influence of noncircular 
motions on the derived rotation curves, allowing for an accurate measurement of all but the inner rotation curve. Consequently, halo parameters, such as $c$, that depend on the entire rotation curve shape can be determined accurately (with a typical uncertainty on $c$ of about $10 \%$ to $20 \%$ ). For DDO 39 and for the new SparsePak data, we found $c$ values that are on the low side when compared to simulations (see Fig. 4).

\section{Conclusions}

The inner slope $\alpha$ is difficult to measure from $\mathrm{HI}$ and $\mathrm{H} \alpha$ long-slit observations because of systematic effects. High-resolution, two-dimensional velocity fields provide a major step forward, but deviations from circular motions still make an accurate measurement of $\alpha$ difficult. A range of inner slopes $0<\alpha<1$ appear consistent with the data, although models with $\alpha=0$ generally provide somewhat better fits. Thus, it seems that an accurate measurement of $\alpha$ and, from that, a tight limit on the nature of dark matter may be difficult to achieve observationally from $\mathrm{H} \alpha$ emission, which is susceptible to kinematic perturbations.

Halo parameters that have been derived from the entire rotation curve instead of only the central parts, such as the halo concentration parameter $c$, may provide a more robust test for cosmological models. In the case of NFW halos, the halo concentration parameters of LSB galaxies tend to be lower than expected from cosmological simulations.

\section{References}

Bell, E. F. \& de Jong, R. S. 2001, ApJ, 550, 212

Blais-Ouellette, S. Carignan, C., Amram, P., \& Côté, S. 1999, AJ, 118, 2123

Bullock, J. S., et al. 2001, MNRAS, 321, 559

de Blok, W. J. G., \& Bosma, A. 2002, A\&A, 385, 816

de Blok, W. J. G., et al. 2001, ApJ, 552, L23

McGaugh, S. S., de Blok, W. J. G. 1998, ApJ, 499, 41

Navarro, J. F., Frenk, C. S., \& White, S. D. M. 1996, ApJ, 462, 563

Navarro, J. F., Frenk, C. S., \& White, S. D. M. 1997, ApJ, 490, 493

Power, C., et al., 2003, MNRAS, 338, 14

Simon, J. D., Bolatto, A. D., Leroy, A., \& Blitz, L. 2003, ApJ, 596, 957

Swaters, R. A. 1999, PhD thesis, Rijksuniversiteit Groningen

Swaters, R. A., Madore, B. F., \& Trewhella, M. 2000, ApJ, 531, L107

Swaters, R. A., Madore, B. F., van den Bosch, F., \& Balcells, M. 2003a, ApJ, 583,732 (SMvdBB)

Swaters, R. A., et al. 2003b, ApJ, 587, L19

van Albada, T. S., Bahcall, J. N., Begeman, K., Sancisi, R. 1985, ApJ, 295, 305

van den Bosch, F. C., Swaters, R. A. 2001, MNRAS, 325, 1017

van den Bosch, F. C., et al. 2000, AJ, 119, 1579

Zentner, A. R. \& Bullock, J. S. 2002, Phys. Rev. D, 66, 43003 